\section{Water Solubility Enhancement of Atorvastatin by Solid Dispersion Method}

${ }^{*}$ Riaz Uddin, Farzana Ali and Subrata Kumar Biswas

Department of Pharmacy, Stamford University Bangladesh, 51 Siddeswari Road, Dhaka-1217, Bangladesh.

\author{
*Corresponding Author \\ Riaz Uddin \\ Lecturer \\ Department of Pharmacy \\ Stamford University Bangladesh \\ 51, Siddeswari Road \\ Dhaka-1217, Bangladesh. \\ Contact no.: +8801749995653 \\ E-mail: kp_ruddin@yahoo.com
}

\begin{abstract}
Atorvastatin is currently used as calcium salt for the treatment of hypercholesterolemia. It is insoluble in aqueous solution of $\mathrm{pH} 4$ and below; it is very slightly soluble in water and $\mathrm{pH} 7.4$ phosphate buffer. In the present study, an attempt was made to enhance the solubility and dissolution characteristics of atorvastatin calcium using solvent evaporation method. HPMC was used as the polymer in different drug to polymer ratios. From the study it was found that HPMC at a drug to polymer ratio of $2: 1$ improves the water solubility of the drug by 2 folds when prepared as solid dispersions by solvent evaporation method.
\end{abstract}

Key words: Solid dispersions, solvent evaporation method, atorvastatin, HPMC.

\title{
INTRODUCTION
}

Most new chemical entities (NCEs) are poorly water soluble drugs, not well-absorbed after oral administration and the oral delivery of such drugs is frequently associated with low bioavailability, high intra- and inter-subject variability, and a lack of dose proportionality (Tang et al., 2007). But due to many advantageous features of this oral rout of drug administration most of the new chemical entities (NCE) under development now-a-days are intended to be used as a solid dosage form that originates an effective and reproducible in-vivo plasma concentration after oral administration (Vasconcelos et al., 2007). To overcome the problems associated with oral absorption and bioavailability issue, various strategies have been utilized including prodrug formation (Murtha and Ando, 1994), complexation (Ghorab and Adeyeye, 2001), microcapsulation (Adeyeye et al., 1994), the use of surfactants, lipids, permeation enhancers, micronization, salt formation, cyclodextrines, nanoparticles, solid dispersions, self emulsifying drug delivery system (Shakhtshneider et al., 1996; Craig, 2002; Gao and Morozowich 2006; Rane et al., 2007; Tang et al., 2007, Uddin et al., 2010) etc. However ahead of all, solid dispersion is the most promising method to the scientists due to the ease of preparation, ease of optimization and reproducibility of the manufacturing method (Chiou and Reigelman, 1971; Ford, 1986; Law et al., 1991; Leuner and Dressman, 2000). Atorvastatin is currently used as calcium salt for the treatment of hypercholesterolemia. It is insoluble in aqueous solution of $\mathrm{pH} 4$ and below; it is very slightly soluble in water and $\mathrm{pH} 7.4$ phosphate buffer. The intestinal permeability of atorvastatin is high at the physiologically relevant intestinal $\mathrm{pH}$. The drug is absorbed more in the upper duodenum and in the upper small intestine regions. However, it is reported that the absolute bioavailability $(F)$ of atorvastatin is $12 \%$ after a $40 \mathrm{mg}$ oral dose (Zhang et al., 2009). In the present study, an attempt was made to enhance the solubility and dissolution characteristics of a poorly soluble model drug, atorvastatin calcium using solid dispersion technology.

\section{MATERIALS AND METHODS}

Experimental material: Atorvastatin calcium was purchased from Dr. Reddy's Laboratory Ltd., India. Hydroxy Propyl Methyl Cellulose (HPMC 6cps) and ethanol were obtained from Samsung, Korea and Merck, Germany respectively. Reagent: Di-sodium hydrogen phosphate and sodium dihydrogen ortho-phosphate (Merck, Germany) and all other ingredients used were of analytical 
grade. Equipment: USP dissolution tester-Apparatus-II (VEEGO, India); UV-VIS Spectrophometer (UV mini-1204, SHIMADZU CORP., Koyoto, Japan); Digital pH meter (pH 211 Microprocessor pH Meter, HANNA Instruments, Romania); Electronic balance (M-310, Denver Instruments, USA); Sieve (Endecott's Test Sieve, Endecotts Limited, England), glass vials, mechanical stirrer, water bath, dessicator etc.

\section{Preparation of the solid dispersion}

Solvent evaporation method (Spenlehauer et al., 1989; Bodmeier and McGinity, 1987) was used to prepare the solid dispersions. Required amount of drug and polymer (Table 1) were mixed in glass vials. Ethanol as solvent was added to the mixture and continuous stirring was ensured for proper mixing of drug and polymer. The mixed mass was kept in an open beaker and the solvent was allowed to be evaporated. The formulations were kept in a dessicator for further treatment. The solidified mass was then crushed, size reduced in a mortar and pestle and sieved through a 150 micron sieve. All glass vials were labeled with care and kept in dessicator. Samples for dissolution studies were taken from the vials.

Table 1: Different ratios of atorvastatin with HPMC.

\begin{tabular}{cccc}
\hline Code & $\begin{array}{c}\text { Atorvastatin calcium } \\
(\mathbf{m g})\end{array}$ & $\begin{array}{c}\text { HPMC 6cps } \\
(\mathbf{m g})\end{array}$ & Atorvastatin calcium:HPMC \\
\hline F-1 & 500 & 125 & $4: 1$ \\
F-2 & 500 & 250 & $2: 1$ \\
F-3 & 500 & 500 & $1: 1$ \\
F-4 & 500 & 1000 & $1: 2$ \\
\hline
\end{tabular}

Physical mixture of atorvastatin calcium and polymer at the same ratio were prepared for comparing the release pattern of the solid dispersions.

\section{Preparation of phosphate buffer $\mathrm{pH} 7.4$}

Phosphate buffer at $\mathrm{pH} 7.4$ was prepared with di-sodium hydrogen phosphate and sodium dihydrogen ortho-phosphet. To prepare one liter of phosphate buffer $1.421 \mathrm{~g}$ di-sodium hydrogen phosphate and $0.227 \mathrm{~g}$ sodium di-hydrogen ortho phosphate were weighed out carefully and dissolved in 1 liter of distilled water. The $\mathrm{pH}$ of the buffer solution was adjusted using a $\mathrm{pH}$ meter.

\section{In vitro dissolution study}

These studies were conducted at a temperature of $37 \pm 0.5^{\circ} \mathrm{C}$ on an USP specification dissolution rate test type II apparatus (Paddle apparatus) with six sections assembly according to the USP 30 procedure (USP 30 and NF 25, 2007). For in vitro dissolution studies, phosphate buffer $\mathrm{pH} 7.4$ was used as dissolution media. Water-bath temperature was fixed \& confirmed to be $37 \pm 0.5^{\circ} \mathrm{C}$ before starting the experiment. The medium was preheated to $37^{\circ} \mathrm{C}$ and then a quantity of $900 \mathrm{ml}$ was added to each vessel. The apparatus was then assembled and paddle rotation was started and adjusted at $100 \mathrm{rpm}$ and the system was allowed to equilibrate for 15 minutes. After that the paddle rotation was stopped and fixed amounts of solid dispersion containing $50 \mathrm{mg}$ equivalent atorvastatin from each batch were placed in the vessels. The apparatus was immediately operated at $100 \mathrm{rpm}$. Each vessel, vessel position and corresponding sample result were assigned the same code. The duration of the experiment was 60 minutes for each set of sample. $10 \mathrm{ml}$ of sample was withdrawn from the media at pre-determined intervals of $5,10,15,20,30,45,60$ minutes. Each and every time $10 \mathrm{ml}$ of dissolution sample was compensated by adding $10 \mathrm{ml}$ fresh phosphate buffer. The sample solutions were diluted and analyzed at $239 \mathrm{~nm}$ for atorvastatin by UV-VIS spectrophotometer. The amount of drug present in the samples was calculated from calibration curve constructed from the standard solution of USP reference standard test drug (Figure 1).

\section{RESULTS AND DISCUSSION}

Effect of HPMC in different drug to polymer ratios on dissolution of atorvastatin can be found in figure 2. It is evident from the current study that the prepared solid dispersions showed better dissolution profile in comparison to raw drug, physical mixtures and atorvastatin with ethanol. Maximum $48.89 \%$ of drug release was attained over the 60 minutes dissolution study in case of 
raw drug and incase of solidified drug after dissolving in solvent (methanol) the \%realease after 60 minutes was $55.61 \%$. Formulation 1 (coded as F-1, drug to polymer ratio $4: 1$ ) and formulation 2 (coded as F-2, drug to polymer ratio $2: 1$ ) showed better dissolution profile $(93.37 \%$ and $95.71 \%$ of drug release in 60 minutes respectively).

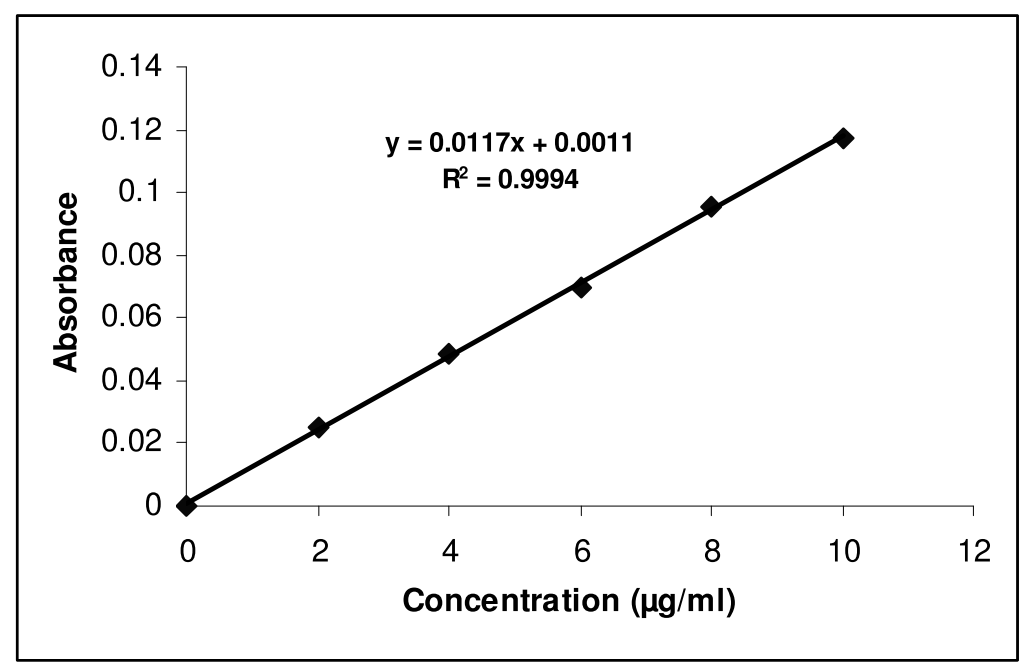

Figure 1: Calibration curve of atorvastatin using phosphate buffer $\mathrm{pH} 7.4$ as media.

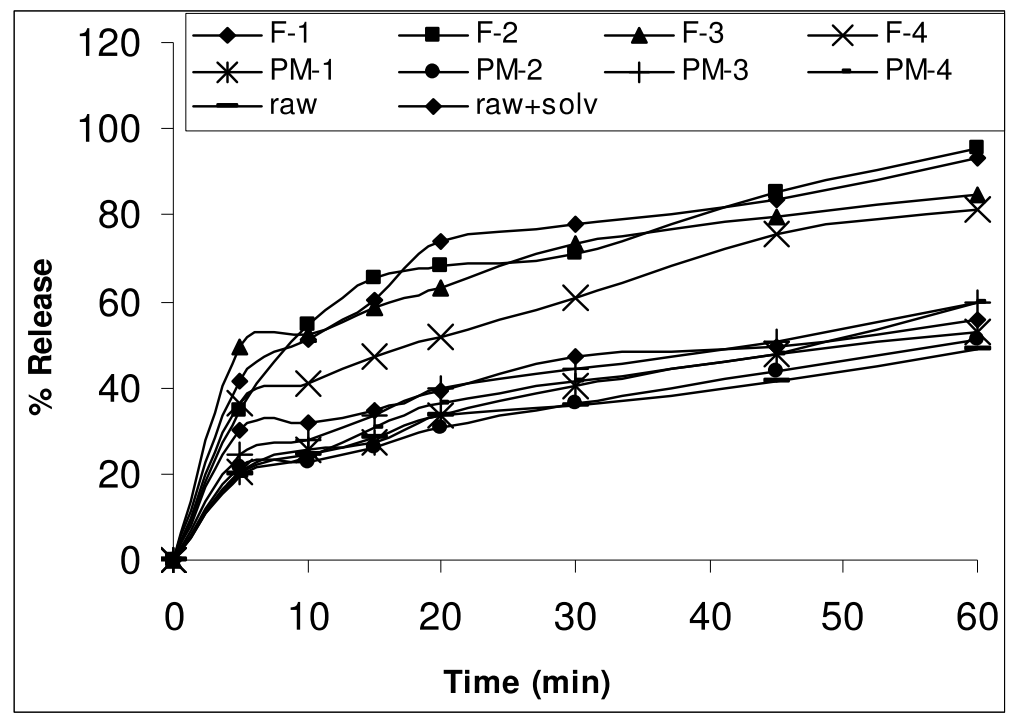

Figure 2: Percent release of atorvastatin from solid dispersions prepared in different polymer ratios of HPMC 6cps ( $F=$ formulation, $P M=p h y s i c a l$ mixture).

Formulation 3 (coded as F-3, drug to polymer ratio 1:1) and formulation 4 (coded as F-4, drug to polymer ratio $1: 2)$ showed improved dissolution time in comparison to raw drug $(85.00 \%$ and $81.13 \%$ respectively over a 60 minutes dissolution study). If we consider the physical mixtures in $4: 1,2: 1,1: 1$ and $1: 2$ drug to polymer ratio $(53.11 \%, 50.96 \%, 59.92 \%$ and $59.57 \%$ of drug release over 60 minutes respectively) we can found that dissolution was improved slightly in comparison to raw drug which indicates that the polymer affect the water solubility and hence dissolution of drug to some extent.

HPMC, a cellulosic polymer showed improved drug release or dissolution profile when solid dispersions were prepared. But from this study no conclusion could be made about the mechanism of enhancement of water solubility. It is also not evident from the data that a higher polymer 
concentration gave better dissolution. In a previous study (Uddin et al., 2010) it was concluded that solid dispersion systems have a potential usage as controlled release drug delivery system. From the current study it is found that increasing drug to polymer ratio may probably decrease the rate of drug release and thus may also be useful in formulating controlled release drugs.

\section{REFERENCES}

Adeyeye CM, Price JC. (1994), Development and evaluation of sustained-release ibuprofen-wax microspheres-II. In vitro dissolution studies, Pharm. Res. 11: 575-579.

Bodmeier R and McGinity JW. (1987), The Preparation and Evaluation of Drug-Containing Poly(dllactide) Microspheres Formed by the Solvent Evaporation Method. Pharmaceutical Research. 4(6):465-471

Chiou WL, Reigelman S. (1971), Pharmaceutical applications of solid dispersion systems. J Pharm Sci. 60(9): 1281-1302.

Craig DQM. (2002), The mechanisms of drug release from solid dispersions in watersolublepolymers. Int. J. Pharm. 231: 131-144.

Ford JL. (1986), The current status of solid dispersions. Pharm Acta Helv. 61:69-88.

Gao P, Morozowich W. (2006), Development of supersaturatable self-emulsifying drug delivery system formulations for improving the oral absorption of poorly soluble drugs. Expert Opin. Drug Deliv. 3(1): 97-110.

Ghorab MK, Adeyeye MC. (2001), Enhancement of ibuprofen, dissolution via wet granulation with beta-cyclodextrin. Pharm. Dev. Technol. 6: 305-314.

Law SL, Lo WY, Lin FM, Chaing CH. (1992), Dissolution and absorption of nifedipine in polyethylene glycol solid dispersion containing phosphatidylcholine. Int. J. Pharm. 84: 161166.

Leuner C, Dressman J. (2000), Improving drug solubility for oral delivery using solid dispersions. Eur J Pharm Biopharm. 50:47-60.

Murtha JL, Ando HY. (1994), Synthesis of the cholesteryl ester prodrugs cholesteryl ibuprofen and cholesteryl flufenamate and their formulation into phospholipid microemulsions. J. Pharm. Sci. 83: 1222-1228.

Rane Y, Mashru R, Sankalia M, Sankalia J. (2007), Effect of hydrophilic swellable polymers on dissolution enhancement of carbamazepine solid dispersions studied using response surface methodology. AAPSPharmSciTech. 8(2): Article 27.

Shakhtshneider TP, Vasiltchenko MA, Politov AA, Boldyrev VV. (1996), The mechanochemical preparation of solid disperse systems of ibuprofen-polyethylene glycol. Int. J. Pharm.. 130: 25-32.

Spenlehauer G, Vert M, Benoit JP, Boddaert A. (1989), In vitro and In vivo degradation of poly(D,L lactide/glycolide) type microspheres made by solvent evaporation method. Biomaterials 10(8):557-563

Tang J, Sun J He ZG. (2007), Self-emulsifying drug delivery systems: strategy for improving oral delivery of poorly soluble drugs. Current Drug Therapy. 2: 85-93.

Uddin R, Saffoon N, Huda NH, Jhanker YM. (2010), Effect of Water Soluble Polymers on Dissolution Enhancement of Ibuprofen Solid Dispersion Prepared by Fusion Method. $S \mathrm{~J}$ Pharm Sci. 3(1): 63-67

United States Pharmacopeia 30 and National Formulary 25. (2007), The United States Pharmacopeial Convention, CD ROM.

Vasconcelos T, Sarmento B, Costa P. (2007), Solid dispersions as strategy to improve oral bioavailability of poor water soluble drugs. Drug Discovery Today. 12 (23/24): 1068-1075.

Zhang H, Wang J, Zhang Z, Le Y, Shen Z, Chen J. (2009), Micronization of Atorvastatin calcium by antisolvent precipitation process. Int. J. Pharm. 374 (1-2): 106-113. 